\title{
An Efficient and Reliable Communication to Reduce Broadcast Storm in VANETs using IMB Protocol
}

\author{
V. Velmurugan ${ }^{\# 1}$ \\ \#1 Agni College of Technology, \\ Chennai.
}

\begin{abstract}
VANET is a number nodes to communicate each other, one node broadcast the message and other node receiving and rebroadcast the message to other vehicle. In this paper, we propose a broadcast protocol IMBP (Intensive Message Broadcast Protocol) transmission on the effective way safety- related information for co-operative driving in vehicular Communication. In this protocol we are using energy aware routing for vehicular networks. Here, addressed three important requirements of ad hoc networks reliability, message redundancy, energy efficiency and node life time. In the design of IMBP, we consider comprehensive research of energy consumed of processing units of transceivers, low no of retransmission allowed per packet, packet size, and the important of Acknowledgement packets. The simulation-based performance evaluation, In IMB protocol is shown higher efficiency, low delay and reliability and packet delivery quality ratio higher than existing approaches for road safety applications.
\end{abstract}

Keywords:- Broadcast storm, message redundancy, IMBP, efficiency, vehicular networks

\section{INTRODUCTION AND MOTIVATION}

VANET has faced many challenges, due to its collision, message redundancy, link unreliability, broadcast storm and hidden terminals. It is also subjected to several issues like waste of bandwidth, energy consumption, power utilisation, unwanted message broadcast in network[1]. Moreover, transmission of same message in the network is major threat to VANET. Thus, intensive message reliability check is essential for improvement in the broadcast protocol in VANET. In this chapter, a novel IMB protocol is proposed for VANET. It performs message reliability checks to confirm the message received from the sender and then uses the vehicle connected list and vehicle eliminating list for transfer of the message. This chapter also discusses the performance of the proposed method in terms of throughput, packet delivery ratio, packet loss ratio and delay.

With improvements in wireless technology, intensive research is being conducted on VANETs. Vehicles should be equipped with GPS system to help sharing of data when vehicular ad hoc network is created and establishment of network configuration and sharing path. Difficulties and challenges faced in ad hoc networks are energy utilization, security, band width limitations, network performance and scalability[2]. A new scheduling algorithm to meet the quality needs of service levels for

\author{
J. Martin Leo Manickam ${ }^{\# 2}$ \\ \#2 St.Joseph's College of Engineering, \\ Chennai.
}

different classes of applications based on the requirements. This methodology has drawbacks seen when the number of nodes and hoop count increases[3]. The data exchange is either structure-based or peer-based. In peer based exchange, the nodes talk to the encountered peers. But, this method has the possibility of message redundancy. The chapter is organised as follows. Section 1 describe the motivation and introduction. In section 2 , literature survey of the broadcasting protocol. In section 3, an overview of proposed IMB protocol has been discussed. This section also explains the vehicle connected list and vehicle eliminating list used in distance routing technique. In section 4 , results of the proposed protocol are presented and discussed and also lists out the parameters is used for VANET simulation. The Conclusion of the proposed method is presented in section 5 .

\section{LITERATURE SURVEY}

The impact of broadcast storm problem finds mention in several papers. But not all of the proposed solutions provide consequences to remedy this problem. The Broadcast Storm Problem may produce a high message overhead, broadcast collision, dissemination postpone, etc. Emergency safety motive requires the message to exceed with none interruption inside the short time period. Emergency protection message dissemination is a time critical occasion, so it is highly crucial to investigate the time parameter even as disseminating the emergency message. Because most of the proposed algorithms had been analyzed for single directional message dissemination schemes, [4] have prompted the suggestion of a brand new Broadcast Storm Suppression Algorithm (BSSA) in a multi directional dual carriageway community scenario. The principal goal in this paper was to suggest a new BSSA that would dynamically adapt the vehicle's location based on the adaptive localization technique to broadcast the emergency safety message based totally on the methodology named as a Selective Epidemic Broadcast (SEB) algorithm. The SEB algorithm reduces the published Broadcast Storm Problem with selecting the automobiles that have dispatched the passive acknowledgment best. The passive acknowledgment suggests the car which wants to communicate with the source vehicle that initiated the transmission. [5] Have proposed a brand new approach referred to as Dynamic Broadcast storm Mitigation 
Algorithm (DBSMA) which may be used to resist the broadcast storm problem in a Vehicular Network (VN).

The implementation of the broadcast message in VANET can also be the reason for several troubles consisting of broadcast storm problem in a dense area network and reliability of broadcast transmission. Thus there are numerous publications published dealing with the accomplishment over those issues. The priority based broadcast scheme can be used for mitigating the storm. In [6] and [7] have proposed the categorization of nodes into multiple instructions which constitute the priority of messages and schedule the messages on the basis precedence. The emergency message has the highest priority. DLAR protocol which is an improvement of the LAR protocol that makes use of vicinity facts to minimize the routing overhead of the ad-hoc network[8]. The ideas of DLAR are based entirely on the following principles. First, all the ones nodes on the same routing path which have the same shifting course and much less pace distinction preserve the path with higher solidity (longer connection time). The second idea is the application of AODV as a route discovery mechanism on top of LAR and picks the next relay node on the basis of the transferring course of the supply node. The third concept is the appointment of the AODV nearby repair mechanism for RREQ message to find the vacation spot node on the disconnected function. The fourth concept is to the restriction of the scope of RREQ broadcast region (request zone) to avoid broadcast storm through decreasing RREQ packet quantity.

Broadcast routing which is generally utilized for information dissemination relating to weather conditions, emergency and warning alerts and road conditions[9]. In this routing method, the packet is sent to all other vehicles in the network using flooding. When message the needs dissemination beyond the radio transmission range, a multihop mechanism is utilized. Thus, in a pure broadcast implementation, all receiving vehicles simply rebroadcast the received messages resulting in a broadcast storm problem. The broadcast storm problem occurs when multiple nodes attempt simulations transmission, causing packet collision and extra delay at Medium Access Control (MAC) layer. A broadcast message is sent only once by using a time to live parameter for avoiding message duplication. But there is degradation in the performance of this routing scheme with the increase in network size. Therefore, designing a broadcast protocol for VANET requires consideration of two major problems, namely, the broadcast storm problem and the disconnected network problem. The disconnected network problem occurs when the number of nodes in the area is not sufficient to help disseminate the broadcast message. The broadcast routing suggested for VANETs defines the following three schemes such as the broadcast problem,[10]Vehicular Broadcast Protocol for Vehicular Ad Hoc Networks (DV-CAST) and Broadcast method for
V2V communication. The urban multi-hop broadcast (UMB) protocol is an 802.11-based solution targeted at reducing the broadcast storm and hidden node problems while maximizing the reliability.

Described several lightweight backbone construction schemes. we use a modified definition from the basic concept, because of its reduced message overhead. [11,12]point out the broadcast storm is minimized only by allowing the farthest vehicle which receives a message to forward it. For this, after successfully receiving a message, vehicles issue a black-burst jamming signal whose duration is directly proportional to the distance between the transmitter and the receiver. There is also a version of the protocol which substitutes repeaters for regular vehicles which cross the intersection, thereby eliminating the need of infrastructure. [13] have proposed a mechanism for dynamic control of the communication range by adjusting the transmission power for mitigation of the effects of the broadcast storm. They have discussed multi-hop broadcasting specially in a shockwave scenario which separates the traffic into two streams with different densities and speeds. When the first vehicle of the following stream meets the last vehicle of the leading stream, it senses the danger and immediately sends a broadcast message to all nearby vehicles as caution to reduce speed.[14] have proposed The Last One (TLO) broadcast method to reduce end-to end delay and broadcast storm problem, and the broadcast storm problem using probability to choose the vehicle for the rebroadcast of an alert message. When a vehicle receives a broadcast message for the first time, the vehicle rebroadcasts the alert message with random probability. This method helps reducing the number of rebroadcasting vehicles and, thereby the broadcast storm problem. This method could not fully ensure avoiding broadcast storm. It just reduced the chances of its occurrence. But this algorithm suffered when GPS provided incorrect information between 1-20 meters. [15] have indicated two possible solutions for mitigating the effects of the "broadcast storm problem", which are to reduce the possibility of rebroadcasts or to differentiate the timing of rebroadcasts. There are five possible schemes proposed by the author to alleviate the broadcast storm problem. First, a probabilistic scheme aims at limiting the number of rebroadcasts.

\section{PROPORSED IMB PROTOCOL}

In IMB protocol, broadcast is an effective way to disseminate information for cooperative routing protocols in VANET. The message of the vehicle is verified by a vehicle connected set and redundancy is inculcated by the vehicle eliminating set distance based scheme. The vehicle is equipped with GPS device. Each vehicle broadcasts the intensive beacon message to its neighbouring vehicles. Beacon message contains intensive message of the sender, time stamp, velocity and vehicle ID. The block diagram of the IMB protocol is in Figure 3.1. 


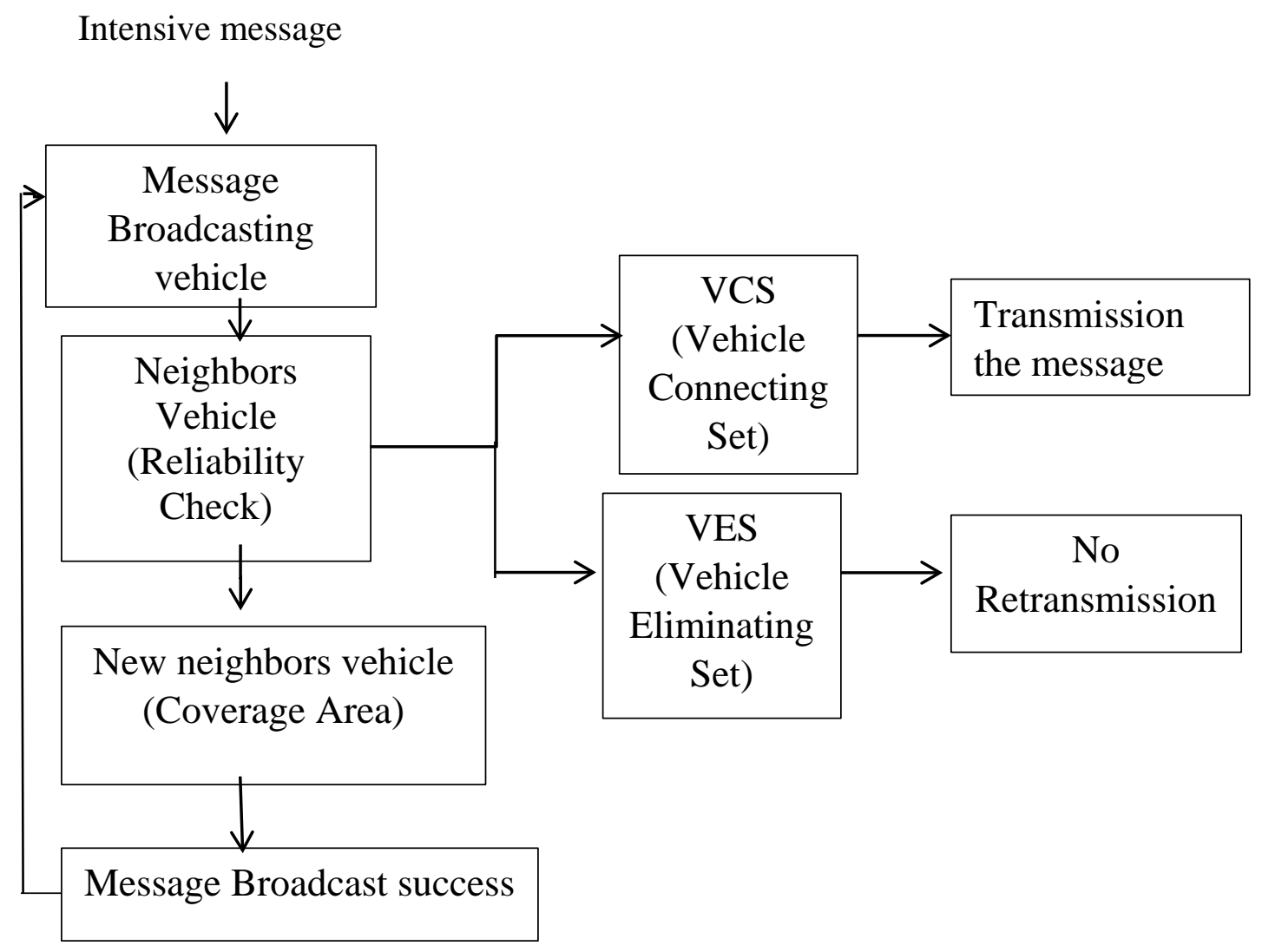

Figure 3.1 Block diagram of IMB protocol

\subsection{Intensive Message}

In IMB protocol, each vehicle broadcasts Intensive messages periodically to its neighboring vehicles. Sharing the information to its neighboring vehicles helps intensive message broadcast and also detect can of the any redundancy message among the set of vehicles. A beacon message is created by the intensive message of the Vehicle. Beacon message contains status information relating to the sender vehicle like speed, position, heading and etc. Beacons provide fresh information relating to the sender vehicle to the nearly vehicles in the network helping them to know the status of the current network and predict the movement of vehicles. A beacon message created by source vehicle is shown in Table 3.1.

Table 3.1 Format of the Message

\begin{tabular}{|l|l|l|l|l|l|l|}
\hline Vehicle ID & Location & $\begin{array}{l}\text { Designation } \\
\text { ID }\end{array}$ & Meta data & Vehicle Speed & Vehicle Direction & Time stamp \\
\hline
\end{tabular}

\subsection{IMB protocol}

IMB protocol is implemented by a Vehicle Connected Set (VCS) and a Vehicle Eliminating Set(VES). While implementing, IMBP contains two lists namely. ' $T$ ' list-the sender and its neighbor with in coverage area and ' $\mathrm{S}$ ' list- which are not connected with the neighbor and also not in coverage area. Initialize the vehicle ' $U$ ' and its Neighbors connected within the network. The flow diagram of the IMB protocol is given Figure 3.2. 


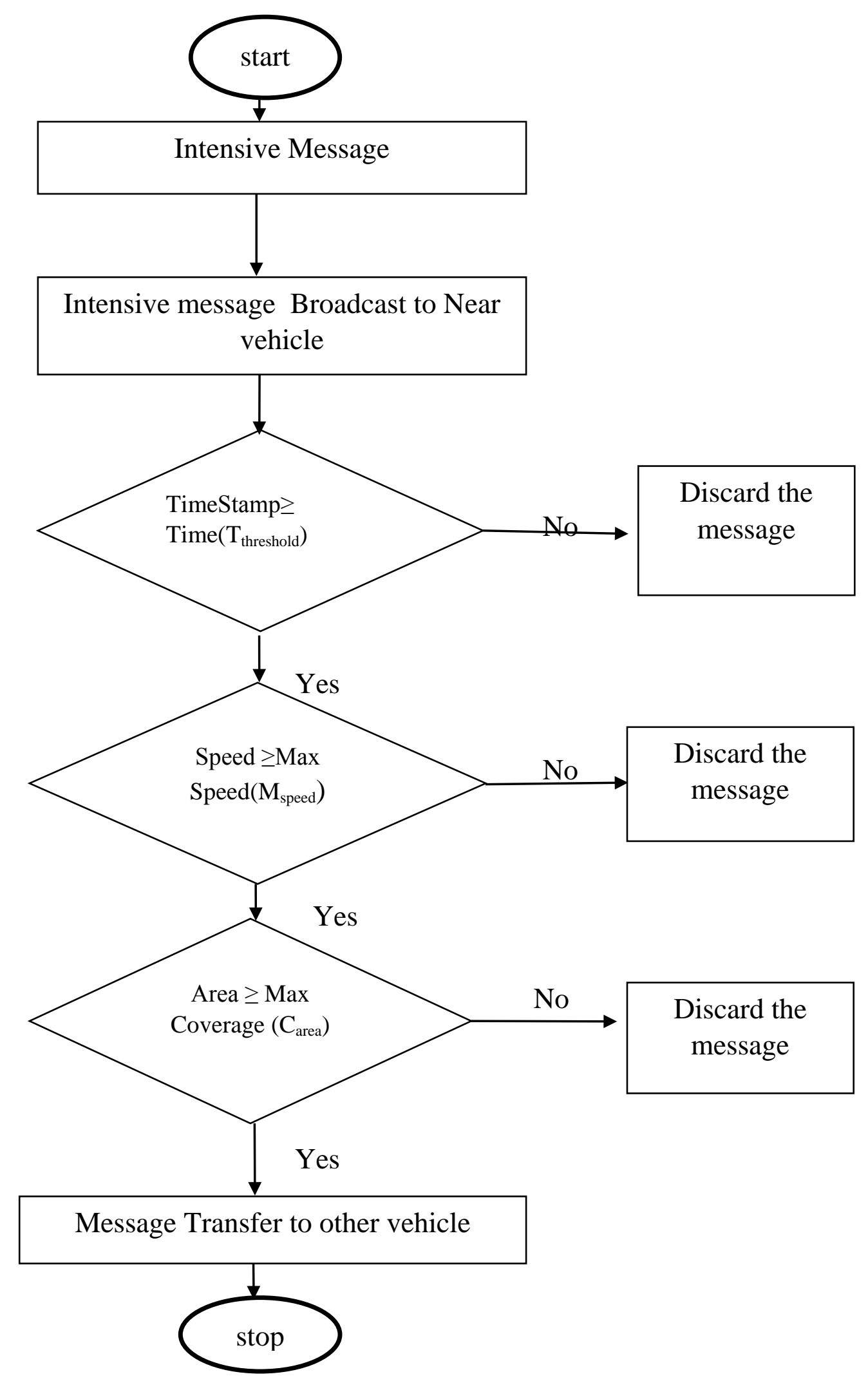

Figure 3.2 Flow chart of IMB Protocol 
Figure 3.2 is the working flow of the IMB protocol. There are two sets, namely, vehicle connected set and vehicle eliminated set. There are six vehicles in the following diagram. The working principle of the IMB protocol is in Figure 3.3.

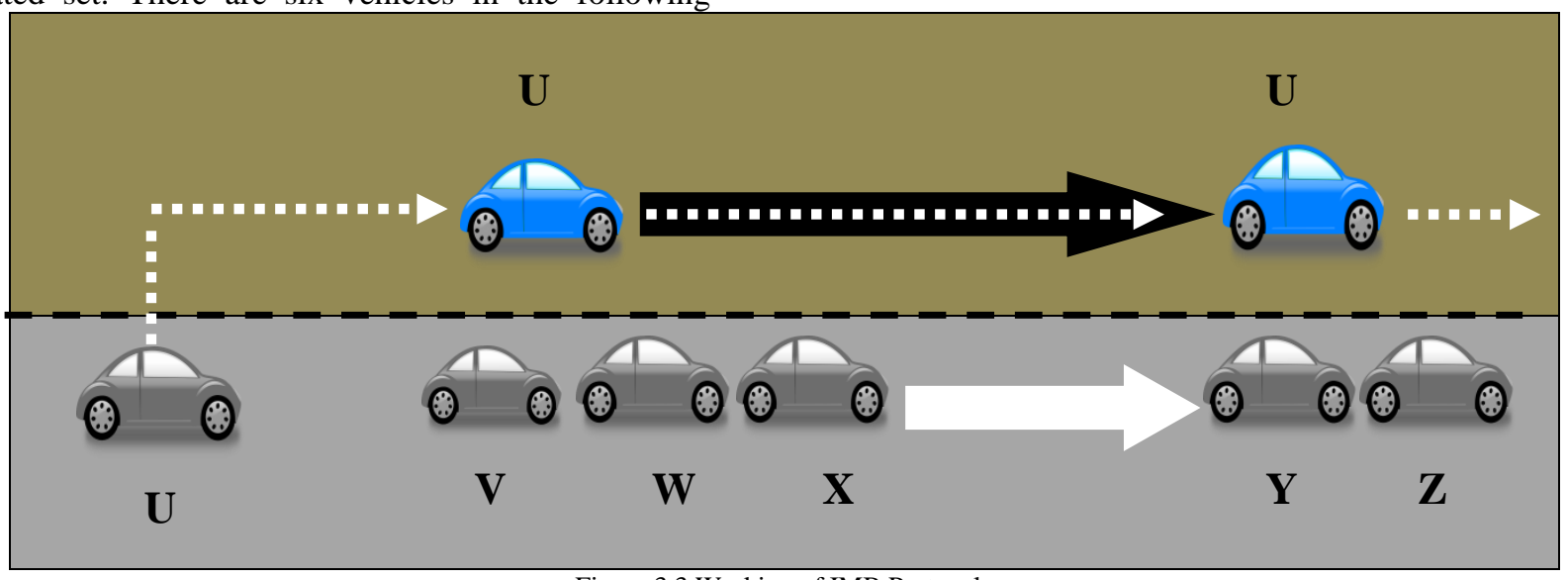

Figure 3.3 Working of IMB Protocol

The vehicles ' $U$ ' to ' $Z$ ' are shown in the above Figure.3.3. Vehicle ' $U$ ' is set to be the sender transmitting an intensive message to its immediate neighbors. When it tries to overtake the nodes $(\mathrm{V}-\mathrm{Z})$, It checks for the node within the VCS for transferring the message. Node ' $\mathrm{V}$ ' and ' $\mathrm{W}$ ' avoid retransmission. Hence, vehicle ' $X$ ' covers both the vehicles. Vehicle 'U' overtakes (Y-Z),'U' vehicle new transmission would occur between ' $Y$ ' and ' $Z$ '. Hence, it has to be covered by node ' $\mathrm{X}$ '. Node ' $\mathrm{Y}$ ' and ' $\mathrm{Z}$ ' have received the message from node ' $\mathrm{X}$ '. Already hence, it is redundant because of node ' $U$ ' covering it again. The node receives an acknowledgement message from node ' $\mathrm{Y}$ ' and ' $Z$ ' (already Intensive message received). So, it discovers the new neighbors but avoids transmitting the message. The following section presents the vehicle connected set.

\subsection{Vehicle Connected Set}

A vehicle performs the reliability check using the speed of the vehicle and timestamp of the vehicle mentioned in the information message received. The difference between the threshold time $\left(\mathrm{T}_{\text {threshold }}\right)$ and the timestamp of received message and the current time is greater than the threshold time. The message is considered to be old and is discarded. Otherwise, the vehicle broadcast the intensive information message to the next hop vehicle. Let, $\mathbf{M}_{\text {speed }}$ be the maximum speed of the vehicle is a given highway area. When the speed of the vehicle moves farther away from $\mathrm{M}_{\text {speed, }}$ it indicates the disobedience of the vehicle in the trouble of the speed regulation in the highway area. Hence, intensive message received from the vehicles is discarded from the transmission. On successful verification of all the reliability check parameters, the intensive message is transmitted to other vehicles. The following section describes the vehicle elimination set.

\subsection{Vehicle Eliminated Set}

A vehicle performs the eliminating check using the coverage area of the vehicle mentioned in the received intensive message. Let $\mathrm{C}_{\text {area }}$ be the maximum transmission range of a vehicle. The distance between the source vehicle and the receiver vehicle is calculated from the signal strength received. If the distance is greater than $\mathrm{C}_{\text {area, }}$, the intensive message is dropped. The reliability check on Coverage area has the ability to identify the location of the sender vehicle within the $\mathrm{C}_{\text {area. }}$ The Vehicle in VES accept the Intensive messages and do not retransmit. It is known through piggyback method, which reduces message redundancy and ensures quicker message transfer from the vehicle.

\section{SIMULATION RESULTS}

In this section, a study of the performance of IMB protocol is made in terms of Packet delivery ratio, throughput, packet loss ratio, delay and control overhead. The IMB protocol is implemented using NS2 version 2.3.4. The performance of IMB protocol is compared with CLB protocol under varying numbers of vehicle densities and varying speed of vehicles. The simulation parameters for implementing IMB protocol are listed in Table 4.1.

Table 4.1 Simulation parameters of IMB protocol

\begin{tabular}{|l|l|}
\hline No. of vehicles & 30 \\
\hline Speed & $5,10,15,20,25$ and $30 \mathrm{~m} / \mathrm{s}$ \\
\hline Media Access Control & 802.11 \\
\hline Transmission Rate & $250 \mathrm{kbps}$ \\
\hline Packet size & 512 bytes \\
\hline Area coverage & $1000 \times 1000 \mathrm{~m}$ \\
\hline Antenna & Omni Directional Antenna \\
\hline Direction & Bidirectional movement \\
\hline Simulation Time & 25 sec \\
\hline Traffic lane & Two lane roads \\
\hline Features of vehicles & GPS enabled vehicles \\
\hline
\end{tabular}


In this topology, there are two lanes in the same direction. Each lane consists of three clusters and each cluster consists of ten vehicles. Hence, there are thirty vehicles in the network, which are placed in an area of $1000 \mathrm{~m} \mathrm{X}$ $1000 \mathrm{~m}$. IEEE 802.11 is used as MAC protocol and the information rate is set at $250 \mathrm{kbps}$. The vehicle which modifies the data before, during or after transmission in the network is referred to as the fast moving vehicle. This provides the correct response with the correct inputs to offer in the network. In the routing mechanism, vehicles are mounted by dropping or correct forwarding of messages in the network. Redundant messages like these relating to routing loops, wormholes and message towards incorrect directions are under in the incorrectly forwarded messages. False location directional transmission, transmission power changes and tracking the vehicle location are classified as redundancy messages. Redundant messages collude for continuous tracking if the location of the vehicle by violating its location privacy. Thus the impact of redundant message is studied for the simulation to analyze the performance of the network.

\subsection{Simulation Parameters}

Throughput: The ratio of the total number of packets successfully sent to the destination to the time allotted is referred as to a throughput which shows a linear increase with the simulation period.

Packet delivery ratio: The ratio of the number of packets successfully delivered to the destination to the total number of packets transmitted is referred to as the Packet Delivery Ratio (PDR) which is linearly increases with the simulation period.
Packet Loss Ratio: The ratio of the number of packets lost at to the destination to the total number of packets transmitted is referred as Packet Loss Ratio.

End - to -End Delay: It is defined as the time taken by the packet to route through the network from the source to its destination node. The average delay can be found by computing the mean of delay of the all message that are sent successfully. Hence, end-to-end delay is contingent on the packet delivery ratio. For example, if the distance between source and destination rises, the probability of packet drop also rises.

Control Overhead: It is defined as the ratio of the total number of control packets generated in the network to the total number of data packets that have reached the destination.

\subsection{Simulation Results and Discussions}

A study of the performance of IMB protocol is made by varying the number of vehicle densities in the network as shown in Figure 3.4. The simulation was carried out by setting the speed of the vehicles at $1 \mathrm{~m} / \mathrm{s}$ to $30 \mathrm{~m} / \mathrm{s}$ with 30 connections. Analysis of the proposed IMBP is done in terms of the packet loss ratio, the packet delivery ratio, throughput, delay and control overhead. The packet delivery ratio of the proposed IMB and existing CLB protocol. The packet delivery ratios of IMB and CLB protocol under varying vehicle densities is in Figure 1.

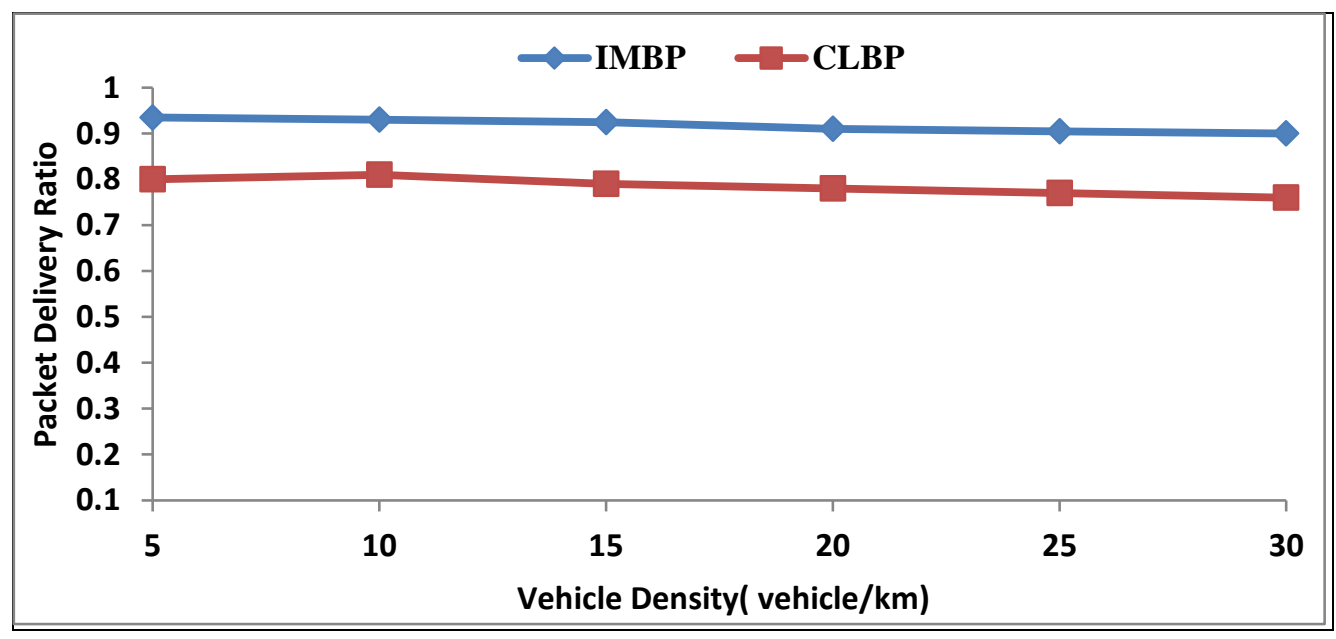

Figure.1 Packet delivery ratio of IMB and CLB protocols under varying vehicle densities

The IMB protocol has the ability to transfer an intensive message in the network effectively. The packet delivery of IMB protocol is 13 percent higher than CLB protocol. Since IMB protocol uses reliability checks in addition to broadcasting message, requests to send and clarity in sending are avoided in the transmission of messages. The simulation results show their ability to deliver a large number of messages during the transmission by implementing the IMB protocol. The Throughput of the proposed IMBP and CLB protocols under varying number of vehicles with vehicle speeds of $1 \mathrm{~m} / \mathrm{s}$ to $30 \mathrm{~m} / \mathrm{s}$ is in Figure 3. 


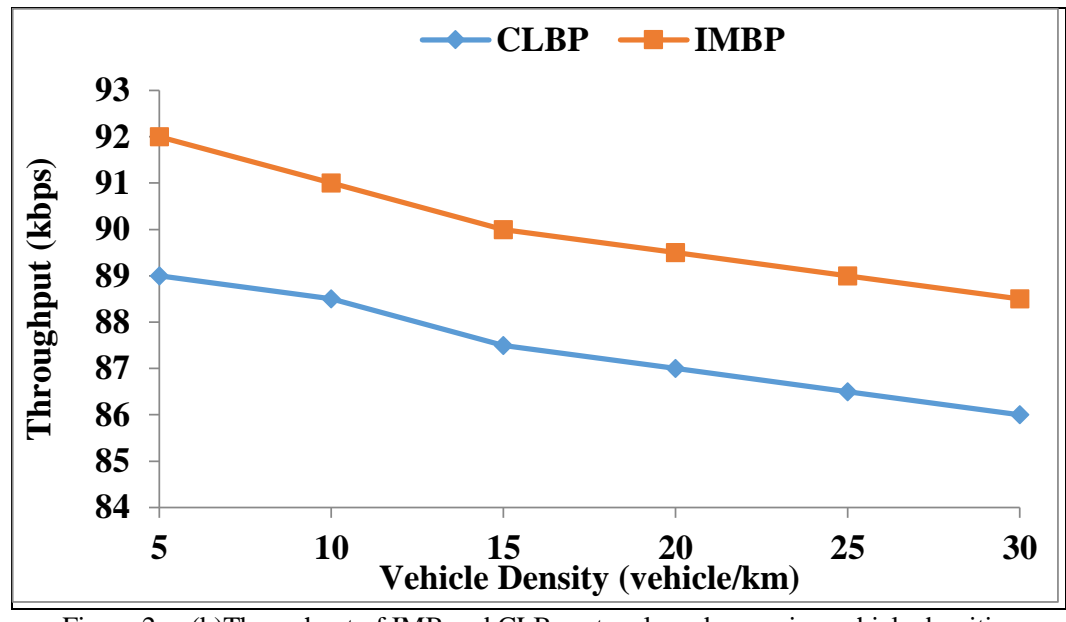

Figure.2 (b)Throughput of IMB and CLB protocols under varying vehicle densities

Figure.2 indicates the throughput of IMB protocol as 18 percent higher than CLB protocol. When the speed of the vehicle increases to $30 \mathrm{~m} / \mathrm{s}$, CLB protocol suffers increased call drop rates and collision rates in the transmission. The
Control overhead of IMB and CLB protocols under varying number of vehicle with vehicle speed of $1 \mathrm{~m} / \mathrm{s}$ to $30 \mathrm{~m} / \mathrm{s}$ is in Figure .3

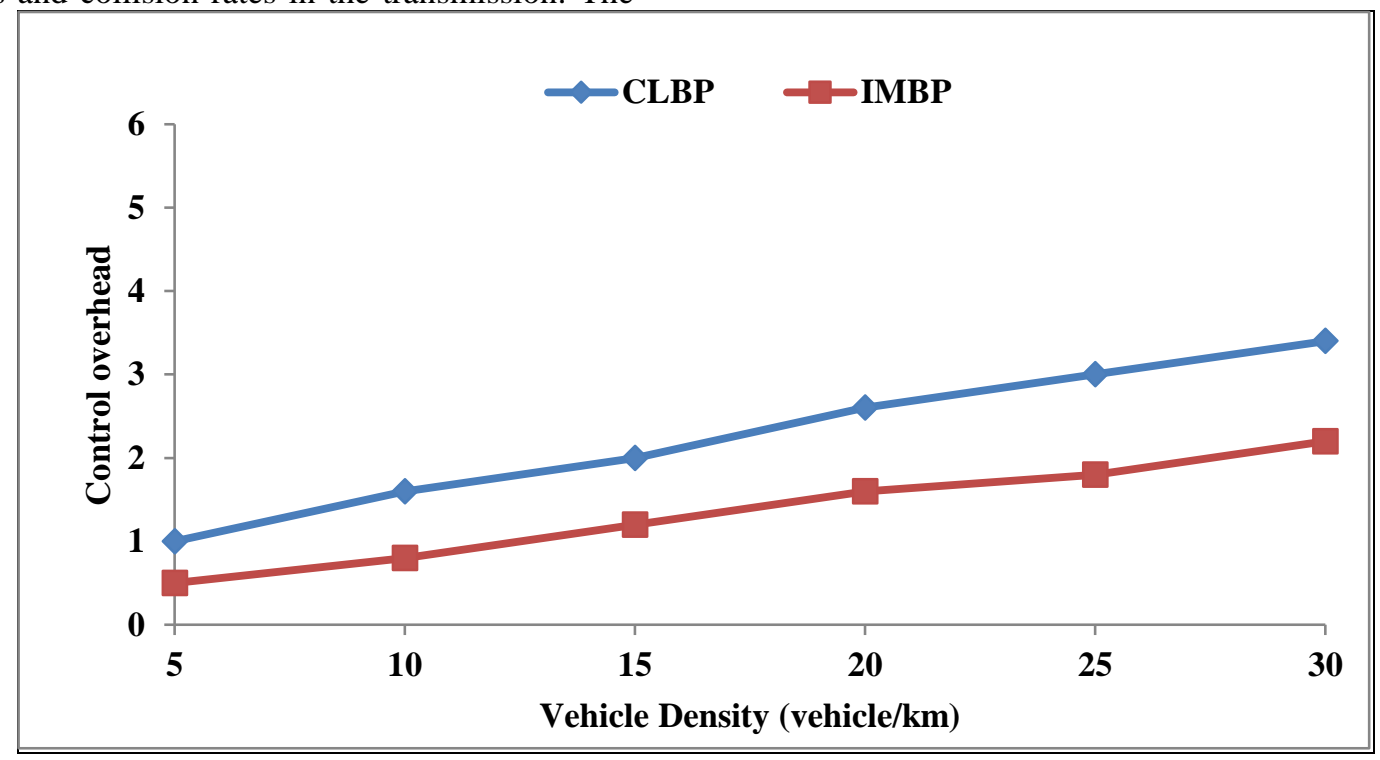

Figure. 3 Control overhead of IMB and CLB protocols under varying vehicle density

Figure. 3 shows the overhead of IMB protocol as 22 percent lower than CLB protocol. However, the speed increases from $5 \mathrm{~m} / \mathrm{s}$ to $30 \mathrm{~m} / \mathrm{s}$. Route changes are made more frequently and route discovery mechanism in CLB protocol is initiated. The results show lower overhead in the IMB protocol due to its use in GPS based location services, control overhead of reestablishing the path when it is distributed due to high mobility of vehicles. The packet loss ratios of IMB and CLB protocol under varying numbers of vehicles with respect to speeds of $1 \mathrm{~m} / \mathrm{s}$ to $30 \mathrm{~m} / \mathrm{s}$ are in Figure 4. 


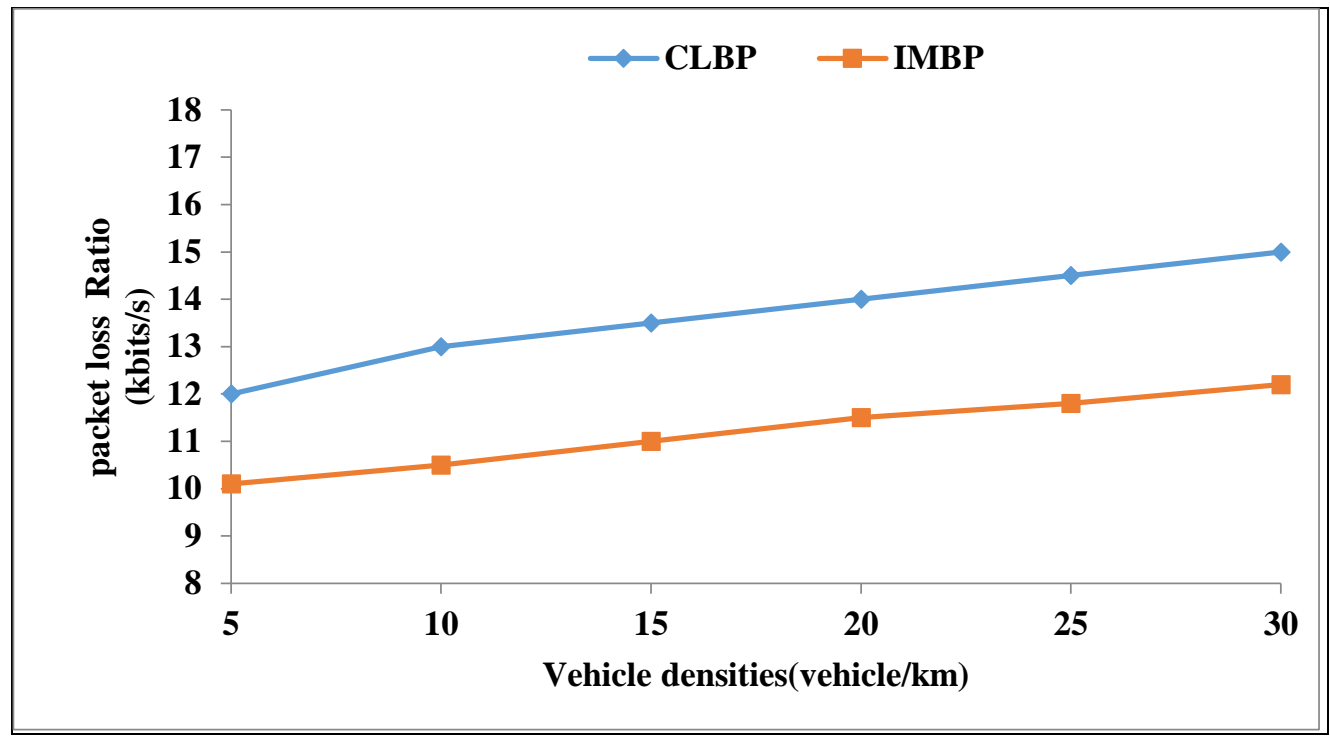

Figure.4 Packet loss ratio of IMB and CLB protocols under varying vehicle densities

The IMB protocol has the ability to identify the location based coverage area in the networks effectively. The packet loss ratio of IMB protocol is 23 percent lower than CLB protocol. When the speed of the vehicles increases, the packet loss ratio decreases. This is due to the cross layer check performed CLB protocol. Hence, the IMB protocol provides effective reliability for the vehicles. As a result, the packet loss ratio of IMB protocol is lower than CLB protocol. The End to End delay of IMB and CLB protocol under varying vehicle densities with vehicle speed $5 \mathrm{~m} / \mathrm{s}$ to $30 \mathrm{~m} / \mathrm{s}$ are in Figure 4.

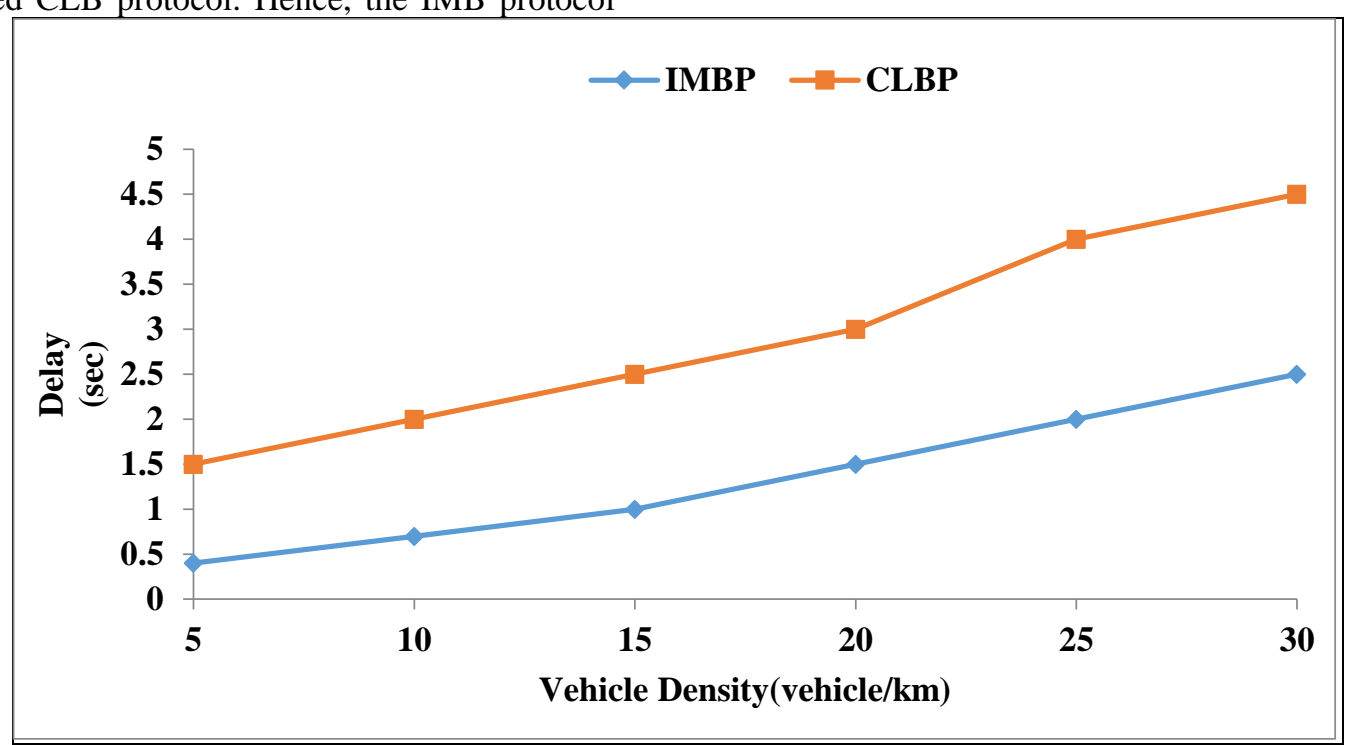

Figure .5 End to End Delay of IMB and CLB protocol under varying the vehicle densities

Figure. 5 indicates the simulation result showing the delay in IMB protocol as 13 percent lower than CLB protocol. Since, CLB protocol performs additional mechanisms such as layer checking and authentication techniques. Hence, the IMB protocol requires lower delay in the network.

The performance of IMB protocol was studied by varying the speed of the vehicles in the network as shown in Figure. 5 Simulation was done by setting by 1 to 30 vehicles in the network. The ratio of PDR is estimated in terms of density which is measured in terms of 4.5 The speed versus PDR,Throughput,Delay, PLR vehicle $/ \mathrm{km}$. The proposed method yields a value from 0.1 to 10 and the speed from $5 \mathrm{~m} / \mathrm{s}$ to $30 \mathrm{~m} / \mathrm{s}$ showing the proposed IMB technique providing a better packet delivery ratio in comparison with the existing technique. The IMB protocol has the ability to do effective transfer of an intensive message in the network. The packet delivery of IMB protocol is 18 percent higher than CLB protocol. Since IMB protocol uses reliability checks in addition to broadcasting message, the request to send and clear to send reply are avoided in the transmission of messages. 


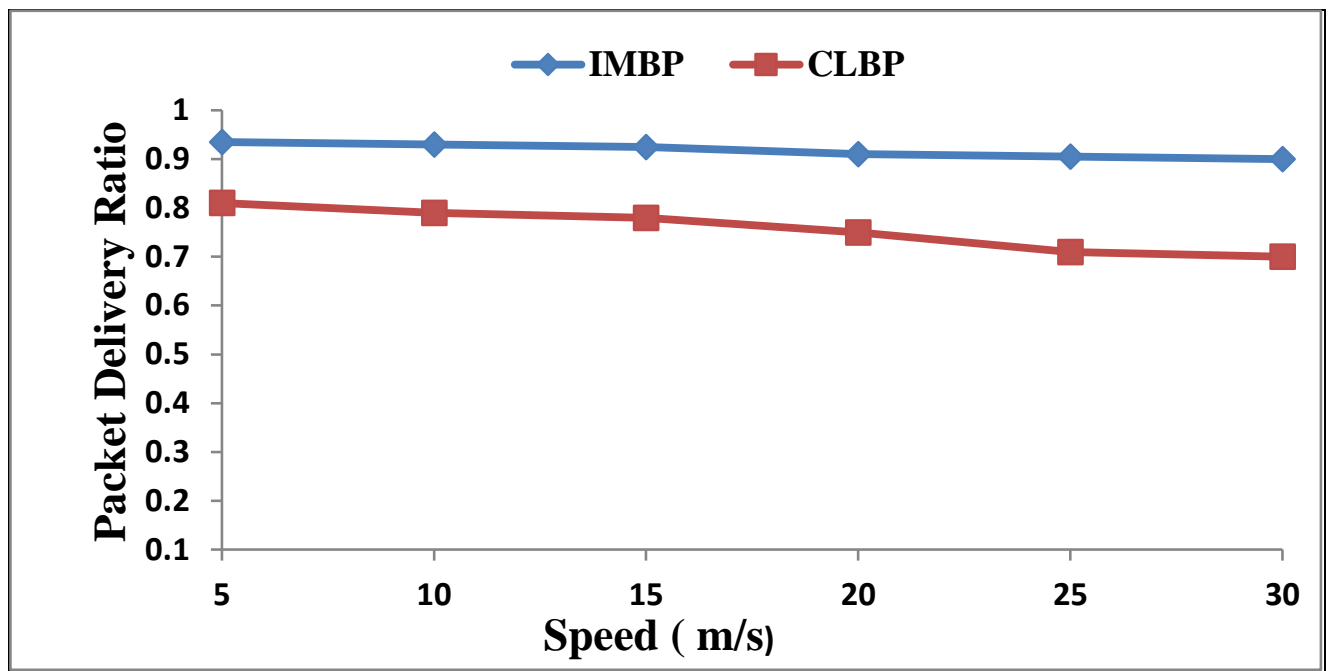

Figure .6 Packet delivery ratio of IMB and CLB protocols under varying vehicle speed

The IMBP protocol has the ability to transfer intensive message in the network effectively. As a result, the packet delivery of IMB protocol 18 percent higher than CLB protocol. Since IMB protocol uses reliability checks in addition to broadcasting message, the request to send and clear to send are avoided in the transmission of messages. The simulation results show the ability to deliver a large number of messages during the transmission.

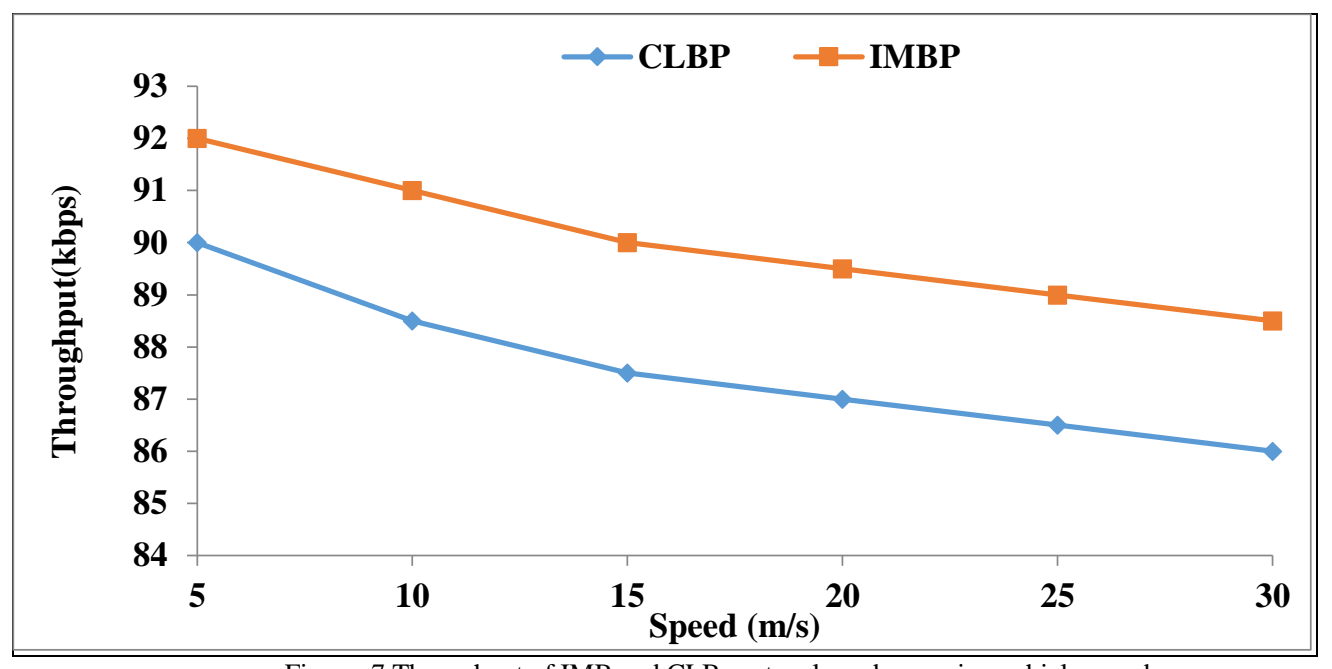

Figure. 7 Throughput of IMB and CLB protocols under varying vehicle speed

Figure.7 indicates the throughput of IMB protocol as 32 percent higher than CLB protocol. When the speed of the vehicle increases to $30 \mathrm{~m} / \mathrm{s}$, CLB protocol suffers increased call drop rates and collision rates in the transmission. The
Control overhead of IMB and CLB protocols under varying numbers of vehicle with vehicle speed of $1 \mathrm{~m} / \mathrm{s}$ to $30 \mathrm{~m} / \mathrm{s}$ are in Figure 7 . 


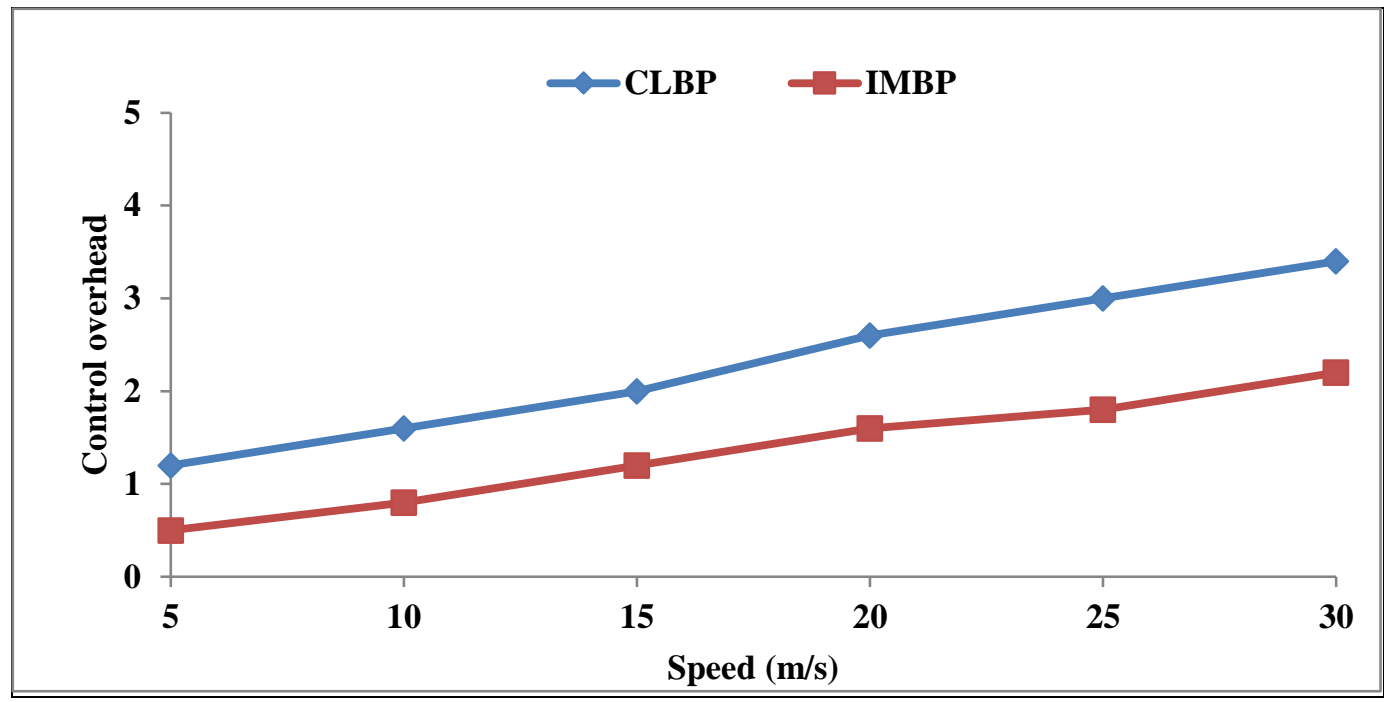

Figure. 8 Control overhead of IMB and CLB protocols under varying vehicle speed

Figure. 8 reveals the overhead of IMB protocol as 22 percent lower than CLB protocol. However, the speed increases from $5 \mathrm{~m} / \mathrm{s}$ to $30 \mathrm{~m} / \mathrm{s}$ route changes are made more frequently and route discovery mechanism in CLB protocol is initiated. The results show lower overhead in the IMB protocol as it uses GPS based location services, control overhead of reestablishing the path when it is distributed due to high mobility of vehicles. The packet loss ratios of IMB and CLB protocol under varying number of vehicles with respect to speeds of $1 \mathrm{~m} / \mathrm{s}$ to $30 \mathrm{~m} / \mathrm{s}$ are in Figure 8. The packet loss ratios of IMB and CLB protocol under varying vehicle speeds are shown in Figure 8.

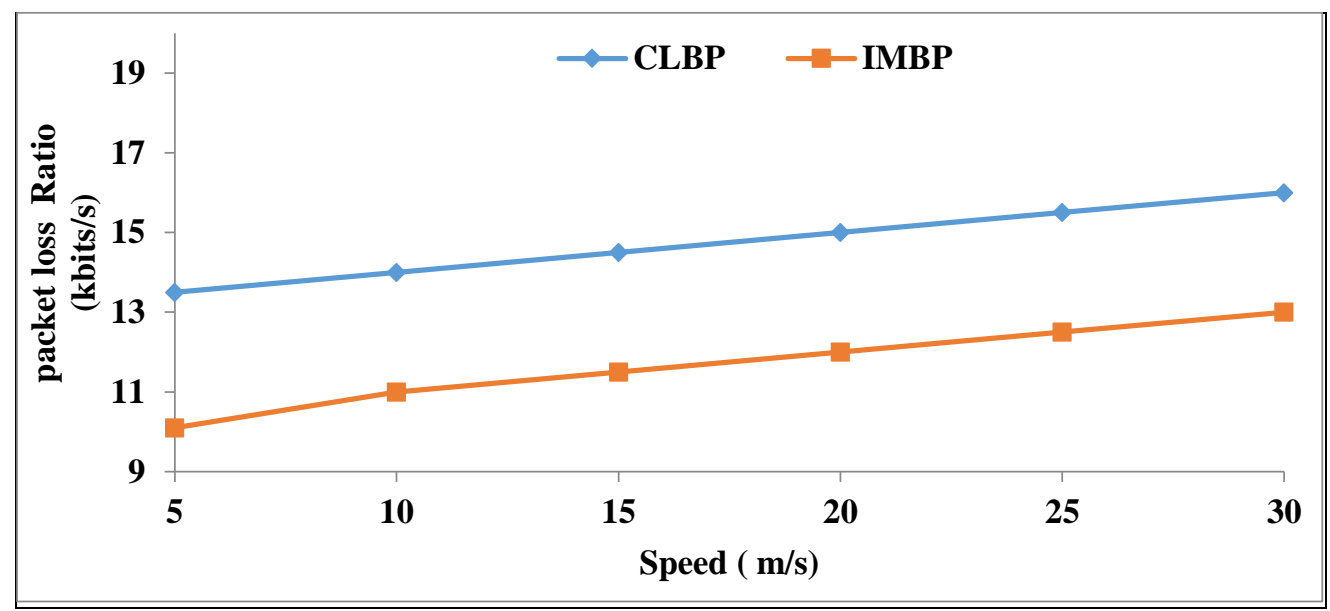

Figure. 9 Packet loss ratio of IMB and CLB protocols under varying vehicle speed

The IMB protocol has the ability to identify the location based coverage area in the networks effectively. The packet loss ratio of IMB protocol is 11 percent lower than CLB protocol. When the speed of the vehicles increases, the packet loss ratio decreases. It is due to the cross layer check performed CLB protocol. Hence, the IMB protocol provides reliability from the vehicles effectively. As a result, the packet loss ratio of IMB protocol is lower than CLB protocol. 


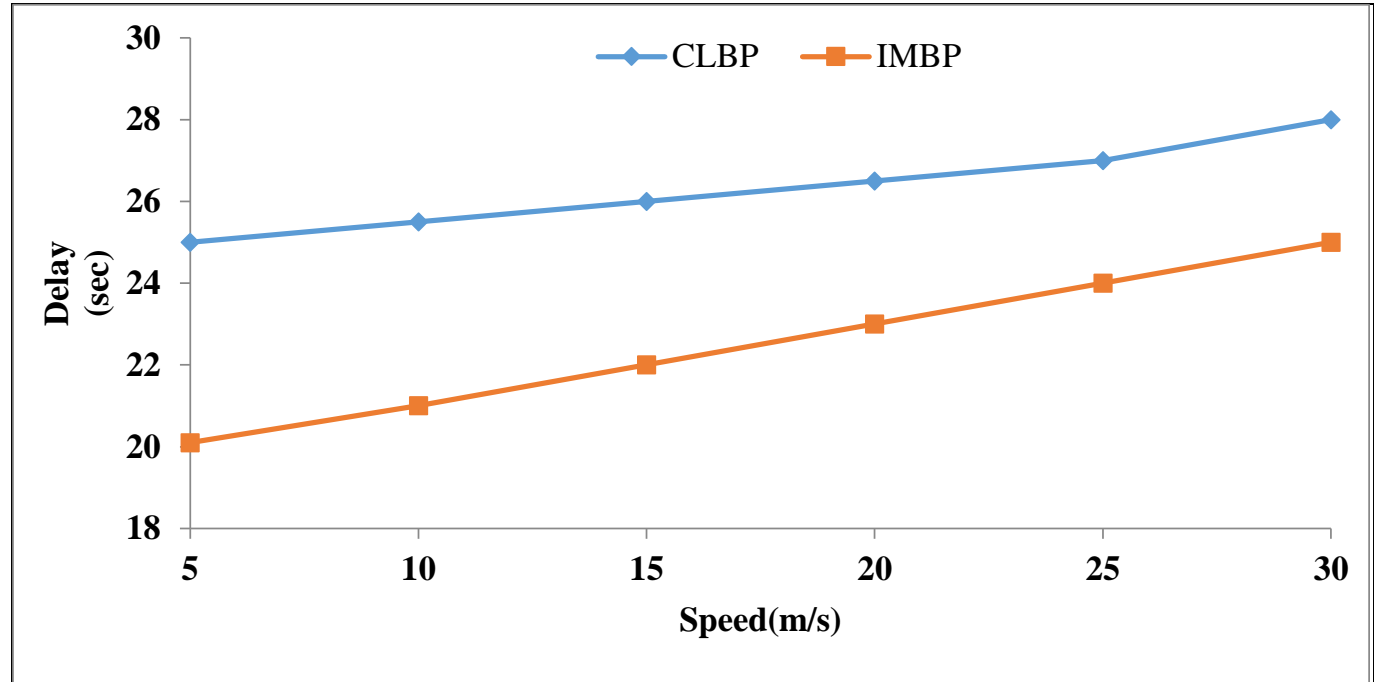

Figure 10.End to End Delay of IMB and CLB protocols under varying the vehicle Speed

Figure.10 indicates the simulation result showing the delay in IMB protocol is 23 percent lower than CLB protocol. Since, CLB protocol performs additional mechanisms such as layer checking and authentication techniques. Hence, the IMB protocol requires lower delay in the network.

\section{CONCLUSION}

In this IMB protocol, has provided a description of the various exiting broadcasting methodologies in the VANET broadcasting protocols and presented a new protocol called IMB protocol. The IMB protocol can be improvised by further increases in the different parameters of the vehicles. The outcome of the system clear to be more efficient in terms of data, throughput, packet delivery ratio, packet loss ratio, delay and control overhead. It also does not able to handle broadcast storm in VANET. Hence, to overcome the above constraints group head node routing protocol is employed. The established IMBP was applied to bring down message redundancy, contention, and collision in V2V transmission of VANET for the inhabited areas. Simulation results show the success the procedure in attaining a smaller packet loss ratio and a higher packet delivery ratio. The anticipated protocol and IMBP are functional in rural and urban scenarios, enabling the association of the performance metrics with the prevailing protocols. The algorithm was improvised by a further increase in the different parameters of vehicles by increasing the simulation throughput value, the number of vehicles in the broadcast and by reducing the transmission packet loss.

\section{REFERENCES:}

[1] Stefan, D, Jonathan, P, Geert, H \& Frank, K, 'Graph-Based Metrics for Insider Attack Detection in VANET Multihop Data Dissemination Protocols', IEEE Transaction on Vehicular Technology, vol. 62, no.4, pp. 1505-1518, 2013.

[2] Esmaeili, T, Mahmood, F \& Hossein, G 2012, 'A Priority based Scheduling Algorithm to Improve Quality of Service from Safety perspective in VANET', Journal of Computing, vol. 4, no.2, pp. 236-239,2012.

[3] Haerri, J, Filali, F \& Bonnet, C, 'Mobility models for vehicular ad hoc networks: a survey and taxonomy', IEEE Communications Surveys \& Tutorials, vol. 11, no. 4, pp. 19-41, 2009.
[4] Chitra M and S. Siva Sathya, 'Selective epidemic broadcast algorithm to suppress broadcast storm in vehicular ad hoc networks', Egyptian Informatics Journal, vol. 19, no. 1, pp. 1-17, 2018.

[5] Francisco, JR, Pedro, MR \& Stojmenovic I, 'AcknowledgmentBased Broadcast Protocol for Reliable and Efficient Data Dissemination in Vehicular Ad Hoc Networks', IEEE Transactions On Mobile Computing, vol. 11, no.8, pp. 33-46, 2012.

[6] Biswas, S Tatchikou, R Dion, F, 'Vehicle-to- Vehicle Wireless Communication protocols for enhancing highway traffic Safety', IEEE Communications Magazine, vol. 44, no.1, pp 7482, 2006.

[7] Khabazian, Mehdi, Sonia Aissa, \& Mustafa Mehmet-Ali, 'Performance modeling of message dissemination in vehicular ad hoc networks with priority.' Selected Areas in Communications, IEEE Journal on 29.1, vol. 9, no.6, pp. 61-71, 2011.

[8] Hung-Chin Jang and Chih-Chia Hung, 'Direction based routing strategy to reduce broadcast storm in MANET', International Computer Symposium, vol. 2, no.3 pp. 445 -450, 2010

[9] Yun-Wei Lin, Yuh-Shyan Chen, \& Sing-Ling Lee, 'Routing protocols in vehicular ad hoc networks: A survey and future perspectives. J. Inf. Sci. Eng., vol. 26, no. 3, pp. 913-932, 2010.

[10] Ozan K Tonguz, Nawaporn Wisitpongphan \& Fan Bai., 'Dv-cast: A distributed vehicular broadcast protocol for vehicular ad hoc networks. Wireless Communications, IEEE, vol. 17, no. 2, pp. 14147,2010

[11] $\mathrm{Wu}, \mathrm{SL}$, Tseng, YC \& Sheu, JP, 'Intelligent medium access for mobile ad hoc networks with busy tones and power control', IEEE J. Sel. Areas Commun., vol. 18, no. 9, pp. 1647-1657, 2000.

[12] Stojmenovic, I, Seddigh, M \& Zunic, J, "Dominating sets and neighbor elimination based broadcasting algorithms in wireless networks", IEEE Transactions on Parallel and DistributedSystems, vol. 15, no. 11, pp. 1054-1055, 2004.

[13] $\mathrm{Wu}, \mathrm{J} \& \mathrm{Li}, \mathrm{H}$, 'A dominating set based routing scheme in ad hoc wireless networks', Telecommunication Systems, vol. 18, no. 1-2, pp. 13-6, 2001.

[14] G Korkmaz, E. Ekici and F. Ozguner, An efficient fully ad-hoc multi-hop broadcast protocol for inter-vehicular communication systems, in Proc. of IEEE International Conference on Communications (ICC), pp. 33-46,2006.

[15] Rex Chen, Wenlong Jin \& Amelia Regan,'Multi-Hop Broadcasting in Vehicular Ad Hoc Networks with Shockwave Traffic', IEEE 7th Consumer Communications and Networking Conference, vol. 9, no.3, pp. 1-5, 2010.

[16] Kanitsom Suriyapaibonwattana \& Chotipat Pomavalai, 'An Effective Safety Alert Broadcast Algorithm for VANET', International Symposium on Communications and Information Technologies, vol. 8, no.5,pp. 247-250, 2008.

[17] Sze-Yao Ni, Yu-Chee Tseng, Yuh-Shyan Chen, Jang-Ping Sheu, 'The Broadcast Storm Problem in a Mobile Ad Hoc Network', Wireless Networks, vol. 8, no. 2, pp. 153-167, 2002. 